\title{
Nanoscale
}

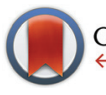

CrossMark \& click for updates

Cite this: Nanoscale, 2016, 8, 19644

\section{Robust surface coating for a fast, facile fluorine-18 labeling of iron oxide nanoparticles for PET/MR dual-modality imaging ${ }^{\dagger}$}

\author{
Ziyan Sun, $t^{\mathrm{a}}, \mathrm{b}$ Kai Cheng, $t^{\mathrm{b}}$ Fengyu Wu, ${ }^{\mathrm{b}, \mathrm{c}}$ Hongguang Liu, ${ }^{\mathrm{b}}$ Xiaowei Ma, ${ }^{\mathrm{b}}$ Xinhui Su, ${ }^{\mathrm{b}}$ \\ Yang Liu, ${ }^{\mathrm{b}}$ Liming Xia*a and Zhen Cheng* ${ }^{* \mathrm{~b}}$
}

Grafting a robust organic shell around inorganic nanoparticles can optimize their colloidal features to dramatically improve their physicochemical properties. Here, we have developed a polymer coating procedure for providing colloidal stability to the nanoparticles and, more importantly, for applying a fast, facile fluorine-18 labeling of iron oxide nanoparticles (IONPs) for positron emission tomography (PET)/ magnetic resonance (MR) dual-modality imaging. The structure of the amphiphilic polymer is based on a backbone of polyacrylic acid, conjugated with multiple oleylamines to form a comb-like branched structure. The dense polymer shell provides high colloidal stability to the IONPs against harsh conditions such as high temperature, low $\mathrm{pH}$ value, and high ion strength. By incorporating a 1,4,7-triazacyclononane (NOTA) chelator to the comb-like amphiphilic polymer for the chelation of aluminum fluoride ions, we applied a one-step radiolabeling approach for a fast, facile radiofluorination of magnetic nanoparticles. The new strategy can significantly reduce the procedure time and radiation exposure. The PET/MR dual modality imaging was successfully achieved in living subjects by using ${ }^{18} \mathrm{~F}$ labeled magnetic nanoparticles.
Received 15th September 2016, Accepted 28th October 2016

DOI: $10.1039 / c 6 n r 07298 d$

www.rsc.org/nanoscale characteristics of radionuclides, decay half-life, and radiolabeling reliability. ${ }^{11}$ Although it would be reliable to monitor the probes to reach the target of interest if the half-life of the radionuclides matches the biological half-life of the probes, the radiation exposure and dose to patients and surgeons need to be taken into consideration especially during the intraoperative use of radiolabeled probes. ${ }^{11}$ Considering the radiation exposure and isotope availability, fluorine-18 labeling is an excellent choice to explore the in vivo behavior of magnetic nanoprobes, to provide quantitative analysis of non-invasive images, and to guide intraoperative margin assessment and evaluation of tumor resection during surgery.

The fluorination of magnetic nanoprobes should be fast, facile and highly efficient. The widely used radiolabeling strategies of nanoprobes usually involve two major processes: surface coating of nanoparticles and chelation of radionuclides. ${ }^{11}$ Although other methods such as the direct doping method could make nanoprobes radioactive, the doped isotopes or materials require direct interference with the crystal structures of nanoparticles, which may not be easily achieved. ${ }^{2,12-14}$ The strong complex binding of radionuclides to the nanoparticles via the chelators of a surface coating is a universal applicable method and can be applied to many different nanoparticles without any changes to the physical and chemical properties of nanomaterials as long as there is a strong interaction between the surface coating and nano- 
particle cores. ${ }^{15}$ However, traditional radiofluorination usually involves multistep conjugations and purifications, sometimes taking several hours to complete. ${ }^{16}$ Recently, a one-step radiolabeling approach based on complex binding of aluminum fluoride $\left({ }^{18} \mathrm{~F}\right.$-AlF) ions using 1,4,7-triazacyclononane (NOTA) chelators has been developed for radiofluorination of many peptides and proteins for targeted PET imaging. ${ }^{16-18}$ This onestep radiolabeling approach avoids several time-consuming conjugation and purification steps, and can be implemented with high efficiency and speed. However, its harsh reaction conditions such as high reaction temperature and low $\mathrm{pH}$ value limit its widespread applications in radiofluorination of temperature- and $\mathrm{pH}$-sensitive molecules or materials. In order to apply a one-step radiolabeling approach for radiofluorination of magnetic nanoparticles, we designed a robust amphiphilic polymer, which not only can make the magnetic nanoparticle water-soluble but can also be chelated with radioactive ${ }^{18} \mathrm{~F}$-AlF ions under harsh reaction conditions. The structure of the amphiphilic polymer is based on a backbone of polyacrylic acid, conjugated with multiple oleylamines to form a comb-like branched structure. The strong hydrophobic interaction between oleylamine coated nanoparticles and hydrophobic side chains is responsible for the formation of a firmly grafted organic shell around the nanoparticles; while multiple carboxylic acid groups of the backbone of the amphiphilic coating make nanoparticles water-soluble. The chelator, 1,4,7triazacyclononane (NOTA), was then conjugated to the comblike amphiphilic polymer for the chelation of aluminum fluoride ions. We used this functional amphiphilic polymer to disperse originally hydrophobic magnetic iron oxide nanoparticles (IONPs) into aqueous solution. The colloidal stability of polymer coated IONPs in a series of buffers with different pHs and ion strengths was investigated in terms of particle size and zeta potential. The thermal stability of surface-coated IONPs in the nearly boiling buffer was studied by measuring their hydrodynamic size and zeta potential. Moreover, we evaluated the physical and chemical properties of surface-coated IONPs after various treatments, especially under harsh labeling conditions. The magnetic properties of labeled nanoparticles were characterized under the same conditions in which the in vivo study was carried out. The relaxivity measurements were performed on both unlabeled IONPs and ${ }^{18} \mathrm{~F}$-AlFlabeled ones as suspension in phantoms. The labeling efficiency was confirmed by measuring the amount of $\mathrm{Al}$ element per nanoparticle when non-radioactive AlF was used as a binding reagent under the same conditions. The radiolabeling process including chelation and purification was further optimized in order to be performed in less than one hour. The radiochemical yield, specific activity, and in vitro stability were measured when radioactive ${ }^{18} \mathrm{~F}$-AlF was used. Finally, we explored the capability of ${ }^{18} \mathrm{~F}$-AlF-labeled magnetic nanoparticles as dual imaging probes for PET/MRI dual modality imaging in living subjects. The PET quantification of ${ }^{18} \mathrm{~F}$-AlF-labeled magnetic nanoparticles in major organs and tissues was performed to investigate their in vivo behaviors such as organ uptake, clearance rate, and in vivo stability. To the best of our knowledge, this is the first work in which a one-step radiolabeling approach was applied for the radiofluorination of magnetic nanoparticles.

\section{Experimental details}

\section{Materials}

Iron pentacarbonyl $\left(\mathrm{Fe}(\mathrm{CO})_{5}\right)$, oleic acid (technical grade 90\%), oleylamine (technical grade 90\%), 1-octadecene (technical grade $90 \%), N, N^{\prime}$-dicyclohexylcarbodiimide, polyacrylic acid (PAA, $\left.M_{\mathrm{w}}=1800 \mathrm{Da}\right)$, aluminum chloride hexahydrate and potassium fluoride were ordered from Sigma/Aldrich. $N$-Hydroxysuccinimide (NHS), $\quad N$-hydroxysulfosuccinimide (sulfo-NHS), and 1-ethyl-3-(3-dimethylaminopropyl)carbodiimide hydrochloride (EDC) were purchased from Pierce Biotechnology. The metal chelator, $S$-2-(4-aminobenzyl)-1,4,7triazacyclononane-1,4,7-triacetic acid ( $p$ - $\mathrm{NH}_{2}$-Bn-NOTA), was obtained from Macrocyclics. Unless otherwise mentioned, all other solvents and chemicals were purchased from Sigma/ Aldrich and used without further purification.

\section{Methods}

The no-carrier-added ${ }^{18} \mathrm{~F}$-fluoride was obtained from an inhouse PETtrace cyclotron (GE Healthcare). The reverse-phase extraction C18 Sep-Pak cartridges were obtained from Waters (Milford, MA, USA) and were pretreated with ethanol and water before use. A syringe filter and polyethersulfone membranes (pore size $0.22 \mu \mathrm{m}$, diameter $13 \mathrm{~mm}$ ) were obtained from Nalge Nunc International (Rochester, NY, USA). Small animal PET scans were performed on a microPET R4 rodent model scanner (Siemens Medical Solutions USA, Inc., Knoxville, TN, USA). The scanner has a computer-controlled bed and $10.8 \mathrm{~cm}$ transaxial and $8 \mathrm{~cm}$ axial field of view (FOVs). It has no septa and operates exclusively in the three-dimensional (3D) list mode. Animals were placed near the center of the FOV of the scanner.

Transmission FTIR spectra in the range of $400-4000 \mathrm{~cm}^{-1}$ were obtained using a Thermo Scientific Nicolet iS50 FT-IR spectrometer. ${ }^{1} \mathrm{H}$ and ${ }^{13} \mathrm{C}$ NMR spectra were recorded on an Agilent-400 NMR spectrometer. Mass spectra of synthetic polymers were recorded by a time-of-flight (TOF) mass spectrometer (AB SCIEX TOF/TOF 5800, Applied Biosystems) equipped with a matrix-assisted laser desorption ionization (MALDI) ion source. A CRC-15R PET dose calibrator (Capintec Inc., Ramsey, $\mathrm{NJ}$ ) was used for all radioactivity measurements. The elemental analyses were performed using an inductively coupled plasma mass spectrometer (ICP-MS, Thermo Scientific Xseries 2 Quadrupole). The nanoparticle samples were suspended in freshly prepared aqua regia (trace metal grade $70 \%$ nitric acid $\left(\mathrm{HNO}_{3}\right): 36 \%$ hydrochloric acid ( $\mathrm{HCl}$ ) (Fisher Scientific), $1: 3 /$ $\mathrm{v}: \mathrm{v})$ and heated until completely dissolved, and then diluted up to $8 \mathrm{ml}$ with double-distilled water. Transmission electron microscopy (TEM) was performed with a FEI Tecnai G2 F20 X-TWIN transmission electron microscope operating at $200 \mathrm{kV}$. Samples were deposited and dried on copper grids covered 
with a Formvar/carbon support film, followed by plasma cleaning. The characterization of sizes of NPs by TEM was performed according to the standard assay protocol (NIST-NCL Joint Assay Protocol, PCC-7), including sample preparation, measurement and result analysis. A minimum of 200 discrete particles were measured from each of at least two widely separated regions of the sample. Version V1.46 of NIH ImageJ was used for image processing, analysis and measurements.

\section{Synthesis of amphiphilic comb-like oleylamine branched polyacrylic acid (COBP)}

Polyacrylic acid (1.8 g) and $N$-hydroxysuccinimide (NHS) were dissolved in $50 \mathrm{~mL}$ of anhydrous $N, N^{\prime}$-dimethylformamide (DMF). 4-Dimethylaminopyridine (DMAP, $101.8 \mathrm{mg}$ ) was added into the above solution. A DMF solution of $N, N^{\prime}$-dicyclohexylcarbodiimide (DCC, $1.72 \mathrm{~g}$ ) was added dropwise into the mixture at $0{ }^{\circ} \mathrm{C}$ over $10 \mathrm{~min}$. The mixture was then stirred at room temperature overnight. Oleylamine $(2.33 \mathrm{~g})$ in $10 \mathrm{~mL}$ of DMF was added dropwise into the above filtrate. The mixture was stirred at room temperature overnight. After filtering the white precipitates, the solvent was evaporated under vacuum. The residue was redissolved in $50 \mathrm{mM}$ sodium carbonate solution $(\mathrm{pH}=9)$. The aqueous solution was purified by dialysis against water $($ MWCO $=1000)$ using a dialysis membrane (Spectrum Laboratories, Inc.). The clear solution was lyophilized, and the resultant white powder was then redissolved in water with a brief sonication. When the $\mathrm{pH}$ of the solution was adjusted to 1 using $1.0 \mathrm{M} \mathrm{HCl}$ solution, the white precipitate appeared. The raw amphiphilic polymer was extracted from the mixture using dichloromethane (DCM). The combined DCM solution was dried over sodium sulfate $\left(\mathrm{Na}_{2} \mathrm{SO}_{4}\right)$, and evaporated under vacuum to produce the final product Comblike Oleylamine Branched Polyacrylic acid (COBP, $M_{\mathrm{w}}: \sim 4000$ $\mathrm{g} \mathrm{mol}^{-1}$ ). The total yield was roughly $40 \%$.

\section{Synthesis of NOTA conjugated COBP (NOTA-COBP)}

To active acid terminals, a solution of amphiphilic COBP (80 $\mathrm{mg}$ ) in $2 \mathrm{~mL}$ of dry dichloromethane (DCM), $N$-hydroxysuccinimide (NHS, $23 \mathrm{mg}, 10 \mathrm{eq}$.$) and 1-ethyl-3-[3-$ (dimethylamino)propyl]carbodiimide (EDC, $38 \mathrm{mg}, 10$ eq.) were added at room temperature and stirred for $4 \mathrm{~h}$. The reaction mixture was then added dropwise into cold diethyl ether/ methanol $(1: 1, \mathrm{v} / \mathrm{v})(10 \mathrm{~mL})$, and the resultant precipitate was centrifuged (5000 rpm for $5 \mathrm{~min}$ ). The supernatant was decanted, and the resulting pellet was further washed (two times). The precipitate of NHS-COBP was dried under high vacuum overnight. The metal chelator, $S$-2-(4-aminobenzyl)-1,4,7-triazacyclononane-1,4,7-triacetic acid ( $p$ - $\mathrm{NH}_{2}$-Bn-NOTA) $(51.7 \mathrm{mg}$, 10 eq.), and diisopropylethylamine (DIPEA) (52.2 $\mu \mathrm{L}, 30$ eq.) were dissolved in $1 \mathrm{~mL}$ of chloroform/DMSO $(5: 1, \mathrm{v} / \mathrm{v})$ and added into the freshly prepared NHS-COBP solution in chloroform $(2 \mathrm{~mL})$. The reaction mixture was stirred at room temperature for $20 \mathrm{~h}$. The resultant mixture was then added dropwise into cold diethyl ether/methanol $(1: 1,10 \mathrm{~mL})$, and the white precipitate was centrifuged ( $5000 \mathrm{rpm}$ for $5 \mathrm{~min}$ ). After the supernatant was decanted, the pellet was further washed and dried under high vacuum to obtain the NOTA-COBP ( $~ 70 \mathrm{mg}$ ). The efficiency of the coupling reaction was confirmed by ${ }^{1} \mathrm{H}$ NMR (Agilent $400 \mathrm{MHz} \mathrm{NMR}, \mathrm{CDCl}_{3}$ ).

\section{Synthesis of $8 \mathrm{~nm}$ iron oxide nanoparticles}

The synthesis of $8 \mathrm{~nm}$ IONPs followed the procedure published previously. ${ }^{19}$ Typically, an iron precursor [iron(III)acetylacetonate, $\mathrm{Fe}(\mathrm{acac})_{3}$ ] (2 mmol, $\left.0.7 \mathrm{~g}\right)$ was dissolved in a mixture of $10 \mathrm{~mL}$ of benzyl ether and $10 \mathrm{~mL}$ of oleylamine. The mixture in the four-neck flask was dehydrated at $110{ }^{\circ} \mathrm{C}$ for $1 \mathrm{~h}$, and was quickly heated to $300{ }^{\circ} \mathrm{C}$ and kept at this temperature for $2 \mathrm{~h}$. After cooling down to room temperature, the product was precipitated by adding $50 \mathrm{~mL}$ of ethanol and collected by centrifugation ( $8000 \mathrm{rpm}$ for $8 \mathrm{~min}$ ). The product was washed with ethanol 3 times. The black-brown product was finally redispersed in $10 \mathrm{~mL}$ of hexane with $0.025 \mathrm{~mL}$ of oleic acid.

\section{Preparation of water-soluble NOTA-COBP-IONPs}

The as-synthesized IONPs $(5.0 \mathrm{mg})$ were dissolved in $1.0 \mathrm{~mL}$ of chloroform containing $13 \mathrm{mg}$ of NOTA-COBP. After the mixture was stirred at room temperature for $30 \mathrm{~min}$, the solvent was slowly evaporated under a nitrogen flow. The residue was then dissolved in $10 \mathrm{~mL}$ of $50 \mathrm{mM}$ sodium carbonate solution after a brief sonication. The soluble nanoparticles were purified with a centrifugal-filter (Amicon Centrifugal filter device, $\mathrm{MWCO}=30 \mathrm{kDa}$ ) and washed three times with deionized water. The purified product (NOTA-COBP-IONPs) was concentrated by the centrifugal-filter and stored at $4{ }^{\circ} \mathrm{C}$. The final product was reconstituted in PBS or water and filtered through a $0.22 \mu \mathrm{m}$ filter for cell and animal studies.

\section{Stability of NOTA-COBP-IONPs (temperature, $\mathrm{pH}$ value and ion strength)}

The colloidal stability of NOTA-COBP-IONPs in aqueous solution was studied by dynamic light scattering (Malvern Zeta Size Nano S-90 DLS instrument). The refractive index of iron oxide was adapted from the Malvern Reference manual (MAN0396 Issue 1.0). The stability of NOTA-COBP-IONPs was validated by measuring the hydrodynamic size distributions and zeta potentials of NOTA-COBP-IONPs in various aqueous solutions. For the stability test at a high temperature, the NOTA-COBP-IONPs (or NOTA-IONPs) in PBS buffer $(1 \times$, ion strength $=170 \mathrm{mM}$ ) were incubated in a $95^{\circ} \mathrm{C}$ water bath for 5 , $10,15,20$, and $30 \mathrm{~min}$. After cooling down to room temperature, the nanoparticle solutions were transferred into cuvettes for DLS measurement. To study the effect of solution $\mathrm{pH}$ on the particle stability, the NOTA-IONPs were incubated in sodium acetate buffers with different $\mathrm{pH}$ values $(3,4,5,6,7,8$, 9 , and 10) for $10 \mathrm{~min}$ before the DLS measurement. The size distributions and zeta potentials of nanoparticles were determined by DLS. Similarly, the NOTA-IONPs were treated with different PBS buffers (at different ion strengths: 17, 34, 85, $170,340,510$, and $850 \mathrm{mM}$ ), and their sizes and zeta potentials were monitored with DLS. 


\section{MR phantom study}

To prove the feasibility of COBP-IONPs as an MRI contrast agent, transverse $T_{2}$-weighted MR images of COBP-IONPs were acquired on a 7.0 T small-animal MRI system (GE Healthcare, USA), with gradient iron concentrations from 0 to $0.6 \mathrm{mM}$. $T_{2}$-Weighted MRI images were acquired on a GE 7.0 T small animal MRI system with the following parameters: $T_{\mathrm{R}}=$ $4000 \mathrm{~ms} ; T_{\mathrm{E}}=40 \mathrm{~ms}$; flip angle $=30^{\circ}$; field of view $(\mathrm{FOV})=$ $6 \times 6 \mathrm{~cm}, 256 \times 256$ matrix; slice thickness $=1 \mathrm{~mm}$. Particles were suspended in $0.5 \%$ agarose gel in $300 \mu \mathrm{L}$ PCR tubes. A series of concentrations of COBP-IONPs were prepared by dilution in $1 \%$ agarose gel and then were solidified in the $300 \mu \mathrm{L}$ vials. The vials were embedded in a home-made holder, which was designed to fit the MRI coil and finally filled with $1 \%$ agarose gel. $T_{2}$ Maps were obtained using a spin echo sequence with the following parameters: $T_{\mathrm{E}}=10,20,40$, $60,80,100$, and $120 \mathrm{~ms}, T_{\mathrm{R}}=4000 \mathrm{~ms}$, FOV $=8 \mathrm{~cm}, 256 \times$ 256 matrix, $1 \mathrm{NEX}$, and slice thickness of $1 \mathrm{~mm}$. The $T_{2}$ quantification was performed by curve-fitting the analytical equation: $M=M_{0} \exp \left(\frac{T_{\mathrm{E}}}{T_{2}}\right)$. All data fittings were performed using a nonlinear least-squares algorithm implemented in the OriginPro 8.1 SP2 (OriginLab Co.) analysis software.

\section{AlF chelation of NOTA-COBP-IONPs}

The non-radioactive labeling of NOTA-IONPs with AlF was performed according to a published protocol on ${ }^{18} \mathrm{~F}$-AlF labeling peptides with minor modifications. ${ }^{20}$ Typically, fifty microliters of $10 \mathrm{mM}$ aluminum chloride solution and $50 \mu \mathrm{L}$ of $20 \mathrm{mM}$ potassium fluoride solution were added into $1 \mathrm{~mL}$ of $100 \mathrm{mM}$ sodium acetate buffer $(\mathrm{pH}=4.5)$. The NOTA-IONPs ( $200 \mu \mathrm{g}$ of $\mathrm{Fe}, 240 \mathrm{pmol}$ of particles) in sodium acetate buffer were added to the above reaction mixture. The reaction mixture was then incubated at $95{ }^{\circ} \mathrm{C}$ for $5 \mathrm{~min}$. After cooling down to room temperature, the product was purified using a size-exclusion column (PD-10) and eluted with PBS into the $1.5 \mathrm{~mL}$ centrifuge vials. The fractions containing AlF-NOTA-IONPs were collected and combined in a sterile vial. The final product was finally concentrated with a spin-filter $(\mathrm{MWCO}=30 \mathrm{kDa})$. The amount of $\mathrm{Al}$ and $\mathrm{Fe}$ in samples was analyzed by ICP-MS.

\section{${ }^{18}$ F-AlF labeling of NOTA-COBP-IONPs}

The radiolabeling of NOTA-IONPs with ${ }^{18} \mathrm{~F}$-AlF was performed according to a published protocol with minor modifications. ${ }^{20}$ Briefly, a QMA Sep-Pak Light cartridge (Waters, Milford, MA, USA) was fixed with approximately $3 \mathrm{GBq}$ of ${ }^{18} \mathrm{~F}$-fluoride and then washed with $2.5 \mathrm{~mL}$ of metal-free water. $\mathrm{Na}^{18} \mathrm{~F}$ was then eluted from the cartridge with $1 \mathrm{~mL}$ saline, from which a $100 \mu \mathrm{L}$ fraction was removed. The $100 \mu \mathrm{L}$ of $\mathrm{Na}^{18} \mathrm{~F}$ in $0.9 \%$ saline $(300 \mathrm{MBq})$ was added into the mixture of $50 \mu \mathrm{L}$ of $100 \mathrm{mM}$ sodium acetate buffer $(\mathrm{pH}=4.5)$ and $3 \mu \mathrm{L}$ of $100 \mathrm{mM}$ $\mathrm{AlCl}_{3}$. The resultant mixture was first reacted in a $1 \mathrm{~mL}$ sealed centrifuge tube at $100{ }^{\circ} \mathrm{C}$ for $15 \mathrm{~min}$. The NOTA-IONPs $(50 \mu \mathrm{g}$ of Fe, 59 pmol of particles) in the same sodium acetate buffer were added to the above reaction mixture. The reaction mixture was then incubated at $95{ }^{\circ} \mathrm{C}$ for $5 \mathrm{~min}$. After cooling down to room temperature, the product was purified using a size-exclusion column (PD-10) and eluted with PBS into $1.5 \mathrm{~mL}$ centrifuge vials. The fractions containing ${ }^{18} \mathrm{~F}-\mathrm{AlF}-$ NOTA-IONPs were collected and combined in a sterile vial. The final product was finally concentrated with a spin-filter $(\mathrm{MWCO}=30 \mathrm{kDa})$.

\section{Stability test of AlF-NOTA-COBP-IONPs}

The release of Al from AlF-NOTA-COBP-IONPs was monitored by ICP-MS. Briefly, the as-synthesized AlF-NOTA-COBP-IONPs $(500 \mu \mathrm{g}$ of $\mathrm{Fe})$ were diluted in $0.5 \mathrm{~mL}$ of PBS buffer $(\mathrm{pH}=7.4)$, and transferred into dialysis membrane tubing (Spectra/Por, MWCO $=3500 \mathrm{Da}$, Spectrum Laboratories, USA). The membrane bag was immersed in $10 \mathrm{~mL}$ of PBS buffer $(\mathrm{pH}=7.4)$ in a $20 \mathrm{~mL}$ scintillation vial and incubated at $37^{\circ} \mathrm{C}$. At the determined time intervals, $0.5 \mathrm{~mL}$ of PBS solution was withdrawn from the scintillation vial and the same amount of fresh PBS was added to keep the total volume constant. The Fe and $\mathrm{Al}$ concentrations of each aliquot were analyzed by ICP-MS.

\section{In vivo small-animal PET imaging}

All animal experiments were performed in compliance with the Guidelines for the Care and Use of Research Animals established by the Stanford University Animal Studies Committee. PET imaging was carried out on a microPET R4 rodent model scanner (manufactured by CTI Concorde Microsystems, Knoxville, TN). ${ }^{5,21,22}$ PET scans were performed at 1-, 2-, and 4-hour post-injection (p.i.) of ${ }^{18} \mathrm{~F}$-AlFNOTA-IONPs via the tail-vein. Typically, each mouse was injected with $4.4 \mathrm{MBq}$ of ${ }^{18} \mathrm{~F}$-AlF-NOTA-IONPs (300 pmol particle per $\mathrm{kg}$ of mouse body weight) in $100 \mu \mathrm{L}$ of PBS via the tail vein $(n=3)$. For the subsequent MR scans, an equivalent amount of ${ }^{18} \mathrm{~F}$-AlF-NOTA-IONPs spiked with NOTA-IONPs (10 $\mathrm{mg}$ Fe per $\mathrm{kg}$ of mouse body weight) was injected intravenously into the mice for both PET and MRI. With the help of a laser beam attached to the scanner, mice were placed in the prone position and near the center of the field of view (FOV) of the scanner. Three min static PET scans were acquired at 1, 2, and $4 \mathrm{~h}$ post injection of ${ }^{18} \mathrm{~F}$-AlF-NOTA-IONPs. During the PET scan, mice were anesthetized with isoflurane ( $5 \%$ induction and $2 \%$ maintenance in $100 \% \mathrm{O}_{2}$ ). Images were reconstructed using a two dimensional ordered subset expectation maximization (OSEM-2D) algorithm. All results were analyzed using ASIPro VM ${ }^{\mathrm{TM}}$ and PET data were expressed as the percentage of the injected radioactive dose per gram of tissue (\%ID per g) based on manual region of interest (ROI) drawing on decaycorrected whole-body coronal and transaxial PET images. Values in three to ten adjacent slices (depending on the size of the tissue or organ) were averaged to obtain a reproducible value of radioactivity concentration. The maximum counts per pixel per minute were obtained from the ROIs and were converted to counts per milliliter per minute using a calibration constant. ROIs were then converted to counts per gram per minute based on the assumption of a tissue density of 1 gram 
per $\mathrm{mL}$, and the image ROI-derived percentage of the injected radioactive dose per gram of tissue (\%ID per $g$ ) value was determined by dividing counts per gram per minute by the injected dose. No attenuation correction was performed.

\section{In vivo MR imaging}

Immediately after PET scans, $T_{2}$-weighted fast spin echo MR images were acquired on a 7.0 T small animal MRI system (GE Healthcare). MRI was performed using the same instrument, protocols, and conditions as in the phantom MRI study. The MRI scans were conducted with a dedicated small-animal MRI scanner, custom-designed pulse sequences and radiofrequency coils. The small-animal scanner consisted of a superconducting magnet (Magnex Scientific) with a $7.0 \mathrm{~T}$ field strength, a gradient (Resonance Research, Inc.) with a clear bore size of $9 \mathrm{~cm}$, a maximum gradient amplitude of $770 \mathrm{mT} \mathrm{m}^{-1}$ and a maximum slew rate of $2500 \mathrm{~T} \mathrm{~m}^{-1} \mathrm{~s}^{-1}$ and a General Electric console and Copley 266 amplifiers. During the MR scans, mice were anesthetized with $2 \%$ isoflurane in oxygen and placed in the prone position. $T_{2}$-Weighted fast spin-echo MR images were acquired on a $7.0 \mathrm{~T}$ small animal MRI system (GE Healthcare) under the following parameters: repetition time $\left(T_{\mathrm{R}}\right)=3000 \mathrm{~ms}, T_{\mathrm{E}}=40 \mathrm{~ms}$, echo train length $=8$, FOV $=$ $4 \times 4 \mathrm{~cm}^{2}$, section thickness $=1 \mathrm{~mm}$, flip angle $=90^{\circ}$. MR images were acquired in both transverse and coronal direction pre-injection and at 4 hours after injection. Transversal and coronal MR images were acquired and the signal intensities were measured in defined ROIs using OsiriX imaging software (OsiriX version 3.2; Apple Computer). The NIH standard was used for tumor imaging processing. The ROI analysis was performed according to the previous publications.

Statistical method. Statistical analysis was performed using the Student's $t$-test for unpaired data. A $p$ value of less than 0.01 was considered significant.

\section{Results and discussion}

\section{Preparation of a NOTA conjugated amphiphilic polymer}

The robust structure of the amphiphilic polymer is based on a backbone of polyacrylic acid (PAA) and consists of two moieties: hydrophobic chains and hydrophilic functional groups. As seen in Fig. 1a, polyacrylic acid $\left(M_{\mathrm{w}} \sim 1800\right.$ Da) was covalently conjugated with multiple oleylamines to form a comb-like branched structure (so-called comb-like oleylaminebranched polyacrylic acid (COBP)). The ratio of oleylamine to polyacrylic acid was optimized in a way that one-third of carboxylic acid groups react with oleylamines, leaving two-third of them intact for further modification with chelators. The selection of a suitable amphiphilic property of COBP for subsequent surface coating was based primarily on finding a balance of the water-solubility of its deprotonated form and the lipid-solubility of its protonated form. We designed a strategy to extract the desired COBPs from the reaction mixture. Typically, the deprotonated or protonated COBPs were selectively extracted with the corresponding solvents from the raw product by adjusting the $\mathrm{pH}$ value of the solution, while the insoluble by-product or precipitate was removed by filtration. The resultant COBP was characterized by the nuclear magnetic resonance (NMR) spectrum and FTIR analysis. The ${ }^{1} \mathrm{H}$ NMR spectra of COBP are shown in ESI Fig. S1. $\dagger$ The signals corresponding to the aliphatic chains were clearly observed at $1.5-2.0 \mathrm{ppm}$. The signals indicating the presence of the amide bonds and alkene groups were seen at 3.0 and $5.3 \mathrm{ppm}$, respectively. The analysis of the ${ }^{1} \mathrm{H}$ NMR spectra provided the evidence that the PAA molecules were covalently conjugated with oleylamine groups. Moreover, the Fourier transform infrared spectroscopy (FTIR) analysis of COBP revealed two strong alkyl stretching peaks at 2920 and $2845 \mathrm{~cm}^{-1}$, demonstrating the presence of grafted oleylamine groups. The $\mathrm{C}=\mathrm{O}$ amide I and II bonds were found at 1623 and $1551 \mathrm{~cm}^{-1}$, respectively, which indicated that oleylamine was successfully grafted on the PAA (Fig. S3 $\dagger$ ).

In order to chelate the AlF ions, the metal chelators, 1,4,7triazacyclononane (NOTA), were incorporated to the backbone of COBP via amide bonds. As seen in Fig. $1 \mathrm{~b}$, the functional chelator containing a free amino group, $S$-2-(4-aminobenzyl)1,4,7-triazacyclononane-1,4,7-triacetic acid ( $p$ - $\mathrm{NH}_{2}$-Bn-NOTA), was linked via amide bonds directly to the carboxylic acid groups of amphiphilic COBP. After purification, the resultant NOTA-COBP was characterized by the NMR spectrum. The ${ }^{1} \mathrm{H}$ NMR spectrum of NOTA-COBP is shown in ESI Fig. S2. $\dagger$ The signals indicating the presence of the aliphatic chains of the NOTA molecules were observed between $2.6-3.6 \mathrm{ppm}$. The signals corresponding to the aromatic rings of the linkages between NOTA and PAA were observed between 7.4-7.7 ppm. The ${ }^{1} \mathrm{H}$ NMR spectrum of NOTA-COBP further confirmed that NOTA molecules were covalently conjugated to the COBP. From the FTIR analysis of NOTA-COBP, the signals corresponding to the aromatic ring bending vibrations were clearly observed in the region of $500-1000 \mathrm{~cm}^{-1}$, indicating the existence of the linkage between NOTA and PAA.

\section{Surface coating process for providing colloidal stability to IONPs}

The ligand-addition coating process is a commonly used strategy to disperse originally hydrophobic nanoparticles in the aqueous solution. Generally, this surface coating process consists of addition of a thin ligand shell over the existing one. As shown in Fig. 1c, the amphiphilic polymers (NOTA-COBP) produce strong hydrophobic interactions with the nanoparticle surfactant (such as oleylamine) and make the nanoparticle cores water-soluble when the hydrophilic groups are deprotonated in the solution. The highly monodisperse IONPs were synthesized according to the previous publications (Fig. 2a). The as-synthesized $8 \mathrm{~nm}$ IONPs coated with an oleylamine surfactant were dispersed and stored in the nonpolar solution. The IONPs were fully mixed with the amphiphilic polymer, followed by slow evaporation, finally leading to the formation of a homogeneous film. The dried-out IONPs were soluble in the slightly-basic solution. The amphiphilic COBP shell around nanoparticles stabilized the monodispersed NPs via the 
a
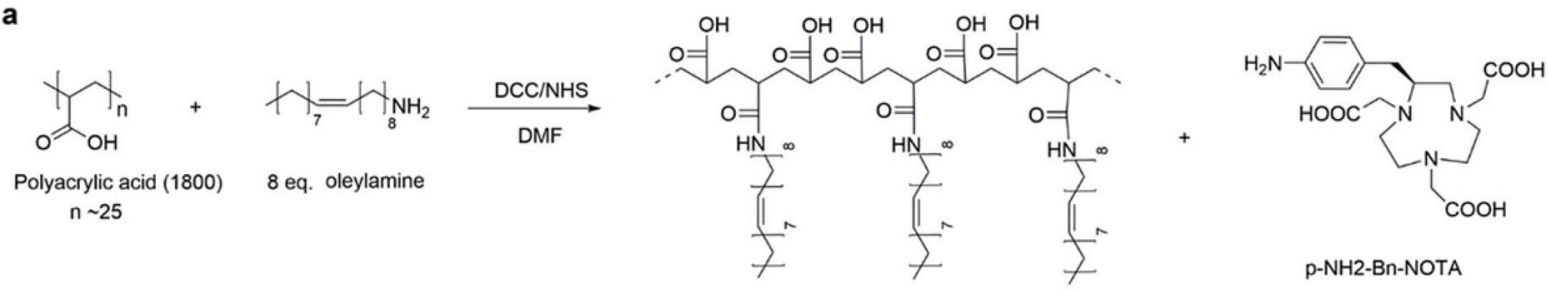

p-NH2-Bn-NOTA

Comb-like Oleylamine Branched Polyacrylic acid (COBP)

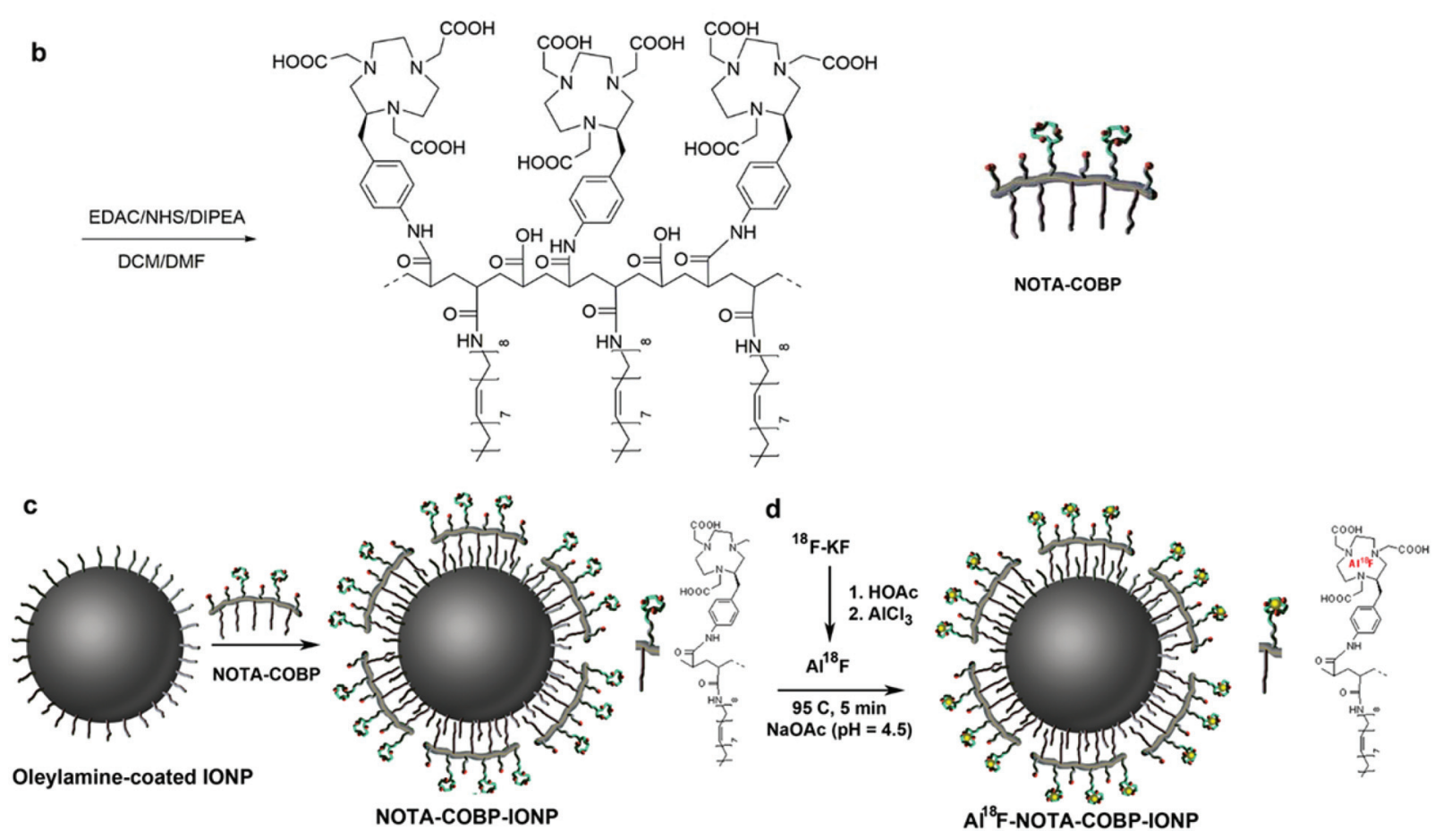

Fig. 1 Schematic illustration of the preparation of water-soluble NOTA-COBP-IONPs (or NOTA-IONPs) and radiolabeling of NOTA-IONPs. (a, b) Preparation of Comb-like Oleylamine-Branched Polyacrylic acid (COBP) and NOTA-conjugated COBP (NOTA-COBP). (a) The branched COBP was synthesized from polyacrylic acid and oleylamine. (b) The conjugation of branched COBP and NOTA via amide bonds. (c) The as-synthesized IONPs $\left(8 \mathrm{~nm}\right.$ in diameter) were coated with a layer of NOTA-COBP molecules via a ligand addition method. (d) The ${ }^{18} \mathrm{~F}$-aluminum fluoride $\left({ }^{18} \mathrm{~F}\right.$-AlF) ions were chelated with the NOTA groups on NOTA-COBP coating of NPs under acidic conditions with a brief heat treatment. The golden balls represent the ${ }^{18} \mathrm{~F}-\mathrm{AlF}$ ions.

surface electrostatic repulsions induced by a dense layer of carboxylic acid groups. More important, the surface coating process including purification steps is fast, facile and straightforward, and can be completed in less than one hour.

\section{Characterization of COBP coated IONPs (COBP-IONPs)}

Size and zeta potential. After IONPs were transferred into the aqueous solution using the COBP polymer, their hydrodynamic size measured by dynamic light scatting (DLS) was $24.3 \pm$ $1.5 \mathrm{~nm}$ in diameter (Fig. 2b). The size of originally hydrophobic IONPs coated with oleylamine in the nonpolar solvent was 15.2 $\pm 1.2 \mathrm{~nm}$. The $9 \mathrm{~nm}$ increase in size was due to the formation of a monolayer of COBP on the particle surface. The deprotonation of a layer of carboxylic acid groups of the COBP coating resulted in a large amount of negative surface charges, which can produce surface electrostatic repulsions to stabilize the nanoparticles in the aqueous solution. The particle zeta potential in water was $-47 \mathrm{mV}$ at room temperature. Because the electrostatic repulsion between nanoparticles depends on the value of zeta potential, the higher zeta potential results in the stronger repulsion, significantly improving the particle stability.

The optical properties of COBP-IONPs under physiological conditions were tested. As seen in Fig. 2c, the as-synthesized IONPs and COBP-coated IONPs were characterized by UV-vis absorbance spectroscopy. By comparing the coated and uncoated particles, the influence of the COBP layer on the optical properties of the IONPs was tested. As expected, both of them were very similar, indicating that the COBP coating did not affect the optical properties of the nanoparticles.

Thermal stability of COBP-IONPs. To evaluate the thermal stability, COBP-IONPs were heated under chelation reaction conditions and their average particle sizes were measured over $30 \mathrm{~min}$. Upon heat treatment, the size and size distribution of COBP-IONPs were monitored by DLS. As shown in Fig. 2d, the 

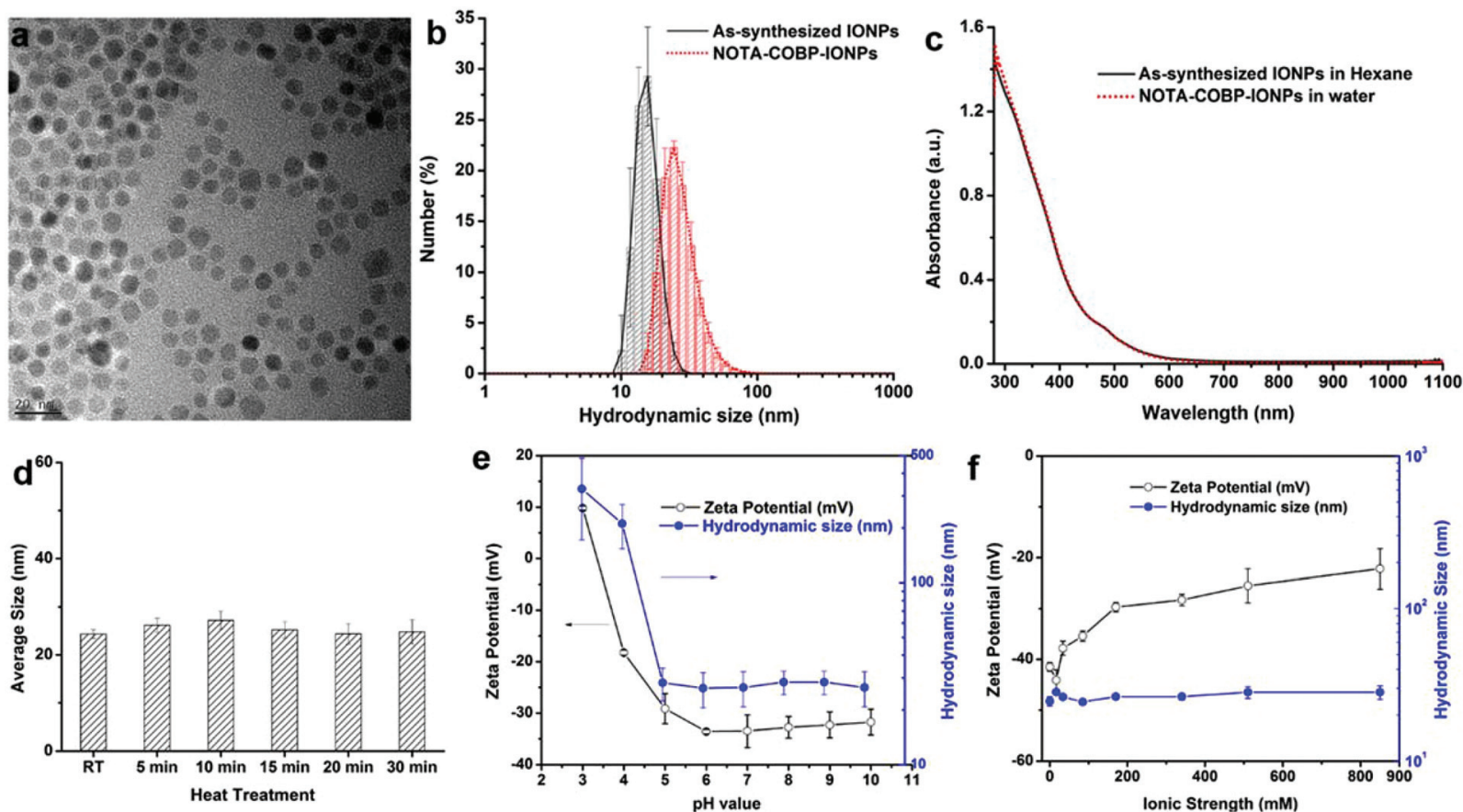

Fig. 2 The surface modification and characterization of NOTA-COBP-IONPs. (a) TEM image of monodisperse iron oxide nanoparticles. (b) The particle sizes and size distributions of as-synthesized IONPs (in hexane) and NOTA-COBP-IONPs (in PBS). (c) UV-vis spectra of as-synthesized IONPs (in hexane) and NOTA-COBP-IONPs (in PBS). (d) The effect of heat treatment on the average size of NOTA-COBP-IONPs in PBS. The particles were heated up to $95^{\circ} \mathrm{C}$ for $5,10,15,20$, and 30 min before cooled down to r.t. (e) The pH-dependent sizes (blue dots and line) and zeta potentials (black circles and line) of water-soluble COBP-IONPs after incubation with buffers at different pHs. (f) lon strength effect on zeta potential (black circles and line) and hydrodynamic size (blue dots and line) of COBP-IONPs after incubation with PBS buffers at different ionic strengths.

average particle size remained nearly constant after heat treatment up to $95{ }^{\circ} \mathrm{C}$ and there was no obvious precipitate in the solution, indicating that the robust COBP coating can tolerate the high temperature treatment.

Effect of $\mathbf{p H}$ on the stability of COBP-IONPs. The effect of the $\mathrm{pH}$ on the stability of COBP-coated IONPs is critical. To study the impact of the solution $\mathrm{pH}$ on the colloidal stability, the particle size and zeta potential of COBP-IONPs were measured in a series of buffers with different pHs. By adjusting the $\mathrm{pH}$ values of buffers from 10 to 3 , the average sizes and zeta potential remained nearly constant until $\mathrm{pH}$ reached 4.5 (Fig. 2e). It is clear that the COBP coated IONPs were stable over a wide $\mathrm{pH}$ range from 4.5 to 10 . Under acidic conditions, however, we observed an obvious pH-responsive behavior for COBP layers, especially for $\mathrm{pH}$ below 4.0. If the $\mathrm{pH}$ of the buffer decreased below the $\mathrm{p} K_{\mathrm{a}}$ of the PAA $\left(\mathrm{p} K_{\mathrm{a}}=4.2\right)$, the carboxyl groups of COBP became protonated, leading to a dramatic decrease in the surface charges. The charge repulsion force induced by the deprotonated carboxyl groups crashed when $\mathrm{pH}$ was below the $\mathrm{p} K_{\mathrm{a}}$ value, eventually resulting in particle aggregation and precipitation. An increase of acidity caused increases of both the zeta potential and particle size when the $\mathrm{pH}$ crossed the $\mathrm{p} K_{\mathrm{a}}$ value.

Effect of ion strength on the stability of COBP-IONPs. To study the impact of ionic strength on colloidal stability, the particle size and zeta potential of COBP-IONPs were measured in a series of PBS buffers with different ion strengths increasing from 0 to $850 \mathrm{mM}$. The average particle size of nanoparticles remained nearly constant, while an increase in the solution ionic strength causes an obvious increase in the particle zeta potential from $-47 \mathrm{mV}$ to $-25 \mathrm{mV}$. It is well known that the charge density around the particle varies with distance. The electrostatic potential decayed exponentially with distance away from the shear plane of the particle. The higher ion strength caused the faster decay and reduced the double layer thickness, thereby resulting in an increase of particle zeta potential. At a high ion strength (i.e. 10× PBS), we found that the electrostatically stabilized colloidal suspensions become unstable and the precipitates appeared in the solution, probably because the double layer collapsed to the extent that the attractive van der Waals forces overcome the charge electrostatic repulsion.

\section{Chelation of AlF ions with NOTA coated COBP-IONPs (NOTA-IONPs)}

To quantitatively measure the number of AlF ions chelated on the NOTA-IONPs, we performed a non-radioactive chelation of AlF and NOTA modified COBP-IONPs under the same chelation conditions. Typically, the NOTA-IONPs were first incubated in different non-radioactive $\mathrm{Al}^{19} \mathrm{~F}$ reaction buffers with different $\mathrm{pH}$ values $(4.0,4.5$, and 5.0). After purification, the particles were digested in acid and the aluminum and iron concentrations were determined by ICP-MS. The number of AlF ions per NP was further normalized by the amount of iron per NP. As shown in Fig. 3a, the chelation efficiency was 


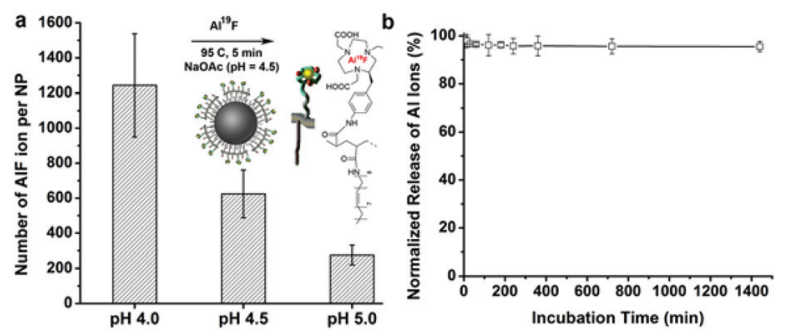

Fig. 3 Quantification analysis of the chelation reaction of aluminum fluoride ions on the NOTA-IONPs. (a) Elemental analysis of AIF-NOTA-IONPs. The NOTA-IONPs were incubated with extra aluminum fluoride $\left(\mathrm{Al}^{19} \mathrm{~F}\right)$ buffer under a brief heat treatment. The $A{ }^{19} \mathrm{~F}$ ions were chelated with the NOTA groups on a NOTA-COBP coating of NPs under acidic conditions. The number of AlF ions per NP was normalized by the amount of iron in the samples. (b) The normalized cumulative release of AlF ions from the nanoparticles, which were incubated with PBS at $37^{\circ} \mathrm{C}$ for 24 hours.

pH-dependent: at $\mathrm{pH}=4$, there were $1243 \pm 294$ AlF ions on a single iron oxide nanoparticle, but the number of AlF ions per $\mathrm{NP}$ at $\mathrm{pH}=5$ was only one quarter of that at $\mathrm{pH}=4$; when the $\mathrm{pH}$ of reaction buffer was 4.5, the number of AlF reached $624 \pm 137$ per NP, which was high enough for radioactive labeling. Considering the chelation efficiency and particle stability at the different $\mathrm{pHs}$, we chose the $\mathrm{pH}=4.5$ reaction buffer for radioactive chelation because of the reasonable labeling efficiency and good particle stability.

To evaluate the stability of AlF chelated NOTA-IONPs in the solution, we measured the cumulative release of AlF ions from the nanoparticles, which were incubated with PBS buffer at $37^{\circ} \mathrm{C}$ in a period of 24 hours. The normalized release of $\mathrm{Al}$ ions from nanoparticles over time is shown in Fig. 3b. There were only about $5 \%$ of $\mathrm{Al}$ ions released from the nanoparticles in the first two hours and there was no further release after that, indicating the high stability of the chelation between AlF and nanoparticles. The released $\mathrm{Al}$ ions from the first two hours were probably due to the dissociation of electrostatically absorbed $\mathrm{Al}$ ions on the nanoparticle surface.

\section{Cytotoxicity of NOTA-IONPs in vitro}

In vitro cytotoxicity of NOTA-IONPs was evaluated via a standard colorimetric assay (tetrazolium dye, MTT) using murine fibroblast cell line NIH-3T3. As seen in Fig. S4, $\dagger$ NIH 3T3 cells showed excellent viability even up to the concentration of $50 \mathrm{mM}$ [Fe] of NOTA-IONPs. Compared to the control (untreated cells), there was no obvious decrease in cell viability of NIH 3T3 cells treated with NOTA-IONPs in the range of 1-50 mM [Fe] for $24 \mathrm{~h}$. Thus, the lack of any noticeable toxicity of NOTA-COBP coated nanoparticles in the given concentration region provided a great opportunity for safe use in molecular imaging and therapy.

\section{Magnetic properties and relaxivity measurement}

To evaluate the magnetic properties of water-soluble magnetic nanoparticles as an MRI contrast agent, we studied $T_{2}$-weighted MR scans of the NOTA-IONPs dispersed in an agar
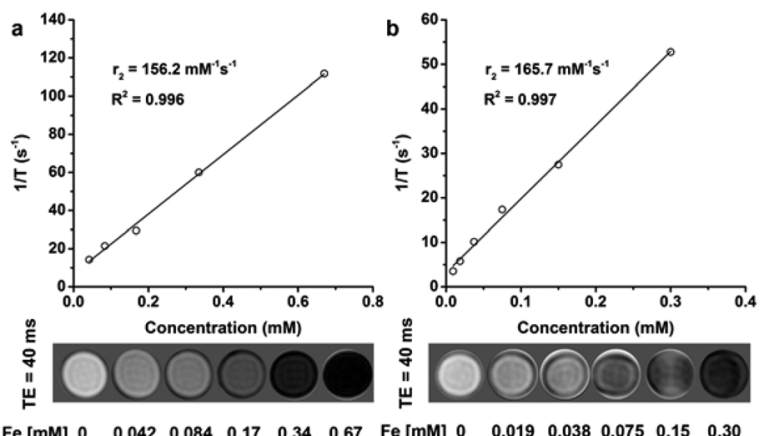

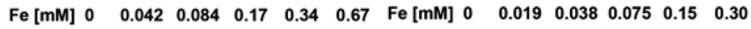

Fig. 4 Magnetic properties of water-soluble NOTA-IONPs and AlFlabeled NOTA-IONPs. (a) $R_{2}$ relaxivity curve of NOTA-IONPs. Relaxivity rate $r_{2}$ was obtained from the slope of the linear fit of the curve of $1 / T_{2}$ vs. Fe concentrations. The phantom images were acquired from $T_{2^{-}}$ weighted MRI scans $\left(T_{\mathrm{E}} / T_{\mathrm{R}}=40 / 4000 \mathrm{~ms}\right)$ for NOTA-IONPs at different iron concentrations $(0,0.042,0.084,0.17,0.34$, and $0.67 \mathrm{mM}[\mathrm{Fe}])$. (b) $\mathrm{R}_{2}$ relaxivity curve of AIF-NOTA-IONPs. The phantom images were acquired from $T_{2}$-weighted MRI scans $\left(T_{E} / T_{R}=40 / 4000 \mathrm{~ms}\right)$ for NOTA-IONPs at different iron concentrations $(0,0.042,0.084,0.17$, 0.34 , and $0.67 \mathrm{mM}[\mathrm{Fe}]$ ).

phantom at a concentration gradient of iron (mM of $\mathrm{Fe}$ ) ranging from $0 \mathrm{mM}$ to $0.67 \mathrm{mM}$. The relaxivity rate $r_{2}$ was obtained from the slope of the linear fit of the curve of $1 / T_{2} v s$. Fe concentrations. As seen in Fig. 4a, NOTA-IONPs can dramatically shorten the $T_{2}$ relaxation of the water molecules with the relaxivity of $156.2 \mathrm{mM}^{-1} \mathrm{~s}^{-1}$, which was very close to that of the commercially available MRI contrast agents (such as Feridex). It suggested that NOTA-IONPs could serve as an effective MR contrast agent. We further investigated the effect of the metal-ion chelation on the magnetic properties. In order to study the magnetic properties of AlF-chelated NOTA-IONPs, we compared the contrast-enhancement effect of the nanoparticles before and after the chelation (Fig. 4b). By graphing changes in relaxation rates at a concentration gradient, the transverse relaxivity of AlF-NOTA-IONPs (165.7 $\mathrm{mM}^{-1} \mathrm{~s}^{-1}$ ) was obtained and remained almost unchanged, suggesting that metal-ion chelation on the particle surface did not change the magnetic properties of iron oxide cores.

\section{In vivo PET imaging and PET quantification}

The radiolabeling process of NOTA-IONPs was very similar to the non-radioactive chelation of AlF and NOTA-IONPs that we described in the previous section. The radiolabeling, including purification and filtration, could be performed within less than one hour. It is very important for ${ }^{18} \mathrm{~F}$ labeling because of the short half-life of ${ }^{18} \mathrm{~F}$. The NOTA-IONPs can be easily labeled with ${ }^{18} \mathrm{~F}$-AlF in a single step with a radiolabeling yield of $40 \%$. Moreover, the specific activity of $1.0 \mathrm{MBq} \mathrm{pmol}^{-1}$ could be obtained. By avoiding several laborious and timeconsuming steps from conventional methods, the radiolabeling process based on the chelation between the ${ }^{18} \mathrm{~F}$-AlF and NOTA-IONPS was fast, facile and highly efficient.

The in vivo behavior of ${ }^{18} \mathrm{~F}$-AlF-NOTA-IONPs was evaluated by PET. Fig. 5a showed the representative coronal and trans- 


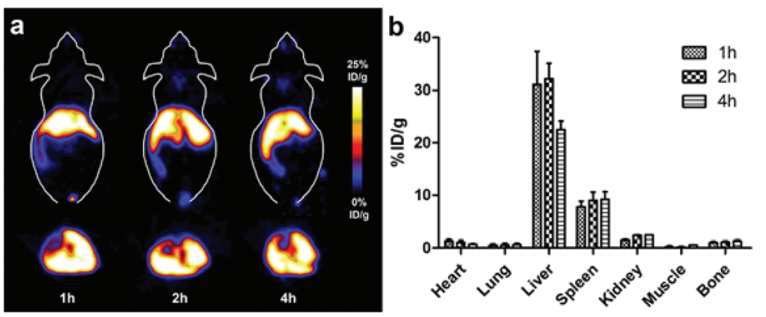

Fig. 5 The ${ }^{18} \mathrm{~F}$ labeled NOTA-IONPs for PET imaging. (a) In vivo PET imaging results of nude mouse at 1,2 and 4 hours after injection with 4.4 $\mathrm{MBq}$ of ${ }^{18} \mathrm{~F}$-AlF-NOTA-IONPs (10 mg Fe per g mouse, $n=3$ ). (b) PET image quantification analysis of ${ }^{18} \mathrm{~F}$-AIF-NOTA-IONPs in major organs and tissues (mean \pm S.D., $n=3$ ).

verse PET images of the nu/nu mice at 1,2, and 4 hours after tail-vein injection of $4.4 \mathrm{MBq}$ of ${ }^{18} \mathrm{~F}$-AlF-NOTA-IONPs. The high uptake in liver and spleen was observed, while there was no bone uptake. On the basis of the quantitative analysis of region of interest (ROI), the nonspecific uptake of nanoparticles by major organs and tissues delineated and localized by the mouse anatomical structure were quantified in the decay corrected PET images (Fig. 5b). The quantification analysis revealed that the liver uptake and spleen uptake were still prominent after $4 \mathrm{~h}$ post-injection $(22.5 \pm 1.6$ and $9.2 \pm 1.5 \% \mathrm{ID}$ per $\mathrm{g}$, respectively), indicating that most of the injected nanoparticles were taken up by the mononuclear phagocyte system (MPS). More important, there was no obvious bone uptake even after 4-hour post-injection. The PET analysis and quantification further confirmed that in vivo behavior and biodistribution of ${ }^{18} \mathrm{~F}$-AlF-NOTA-IONPs were favorable for the molecular
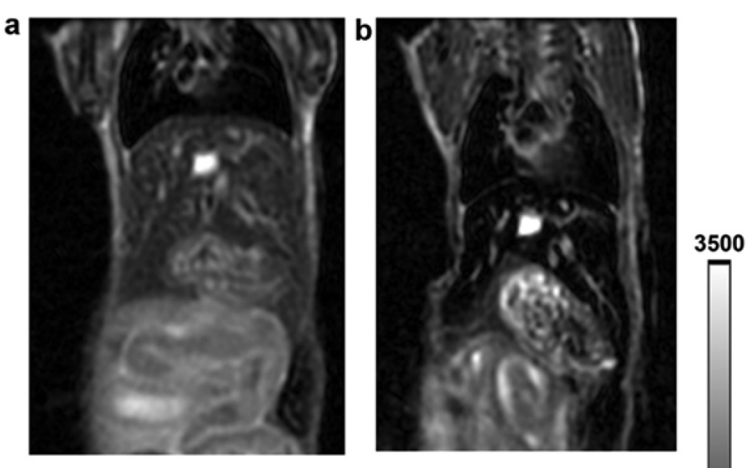

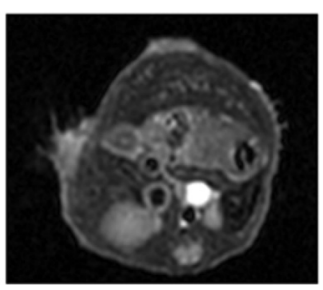

Prescan

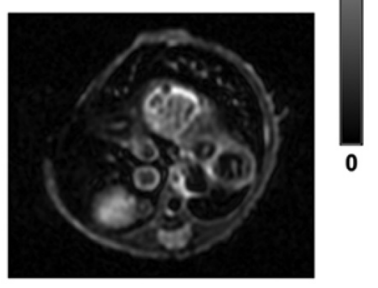

4h postscan
Fig. 6 In vivo $T_{2}$-weighted $M R$ images of nude mice (a) before and (b) $4 \mathrm{~h}$ post injection of ${ }^{18} \mathrm{~F}$-AIF-NOTA-IONPs. ${ }^{18} \mathrm{~F}$-AIF-NOTA-IONPs (4.4 $\mathrm{MBq}$ in $150 \mu \mathrm{L}$ of PBS) were injected via the tail vein into the nude mice at a dose of $10 \mathrm{mg}$ Fe per $\mathrm{kg}$ of mouse weight $(n=3)$. imaging in the living subjects. It is important to make the coated nanoparticles targetable for in vivo application, therefore able to reach the pathological sites specifically. The ongoing research mainly focuses on the modification of the nanoparticles with targeting moieties to reduce the MPS uptake and enhance the targeting efficiency.

\section{In vivo MR imaging}

To investigate the capability of ${ }^{18} \mathrm{~F}$-AlF-NOTA-IONPs as contrast agents for dual-modality $\mathrm{PET} / \mathrm{MR}$ imaging, the in vivo $T_{2}$-weighted fast spin-echo MR scans were performed with the same mice immediately after their PET scans. MRI scans were performed at 4-hour post-injection of ${ }^{18} \mathrm{~F}$-AlF-NOTA-IONPs, which were spiked with NOTA-IONPs. The representative $T_{2}$-weighted MR coronal and transverse images of the mouse prior to and after injection of nanoprobes are shown in Fig. 6. The hypointense signal in the liver was clearly observed after $4 \mathrm{~h}$ post-injection of magnetic nanoprobes, which correlated well with the corresponding PET images. With high spatial resolution, the MR imaging provided the detailed anatomic information and probe distribution.

\section{Conclusion}

In conclusion, we developed a fast, facile surface coating procedure to apply a one-step ${ }^{18} \mathrm{~F}$ radiolabeling of iron oxide nanoparticles for in vivo dual PET/MR imaging. As a universal applicable method, the surface coating procedure can provide high colloidal stability to most of the originally hydrophobic nanoparticles regardless of their size, shape, and composition. The robust surface coating can stabilize the iron oxide nanoparticles against harsh reaction conditions such as high temperature, low $\mathrm{pH}$ value, and high ion strength. With the help of a robust surface coating, one-step ${ }^{18} \mathrm{~F}$ radiolabeling based on complex binding between AlF ions and NOTA groups was successfully applied for radiofluorination of magnetic nanoparticles. Moreover, the new strategy can significantly reduce the procedure time and radiation exposure. The PET/MR dual modality imaging was successfully achieved in living subjects by using ${ }^{18} \mathrm{~F}$ labeled magnetic nanoparticles. The complementary and synergic information obtained from dual PET/MR imaging probes can significantly improve diagnostic performance for disease detection. Therefore, this surface coating strategy could significantly reduce the procedure time and radiation exposure, and have a great potential for the construction of nanoparticle-based multimodality imaging probes for cancer detection.

\section{Acknowledgements}

This work was supported, in part, by the Office of Science (BER), U. S. Department of Energy (DE-SC0008397), NCI of Cancer Nanotechnology Excellence Grant CCNE-TR U54 CA119367, CA151459, NIH In vivo Cellular Molecular Imaging 
Center (ICMIC) grant P50 CA114747, and the National Natural Science Foundation of China 81471637.

\section{References}

1 M. A. Pysz, S. S. Gambhir and J. K. Willmann, Clin. Radiol., 2010, 65, 500-516.

2 Q. Liu, Y. Sun, C. Li, J. Zhou, C. Li, T. Yang, X. Zhang, T. Yi, D. Wu and F. Li, ACS Nano, 2011, 5, 3146-3157.

3 J. Xie, K. Chen, J. Huang, S. Lee, J. Wang, J. Gao, X. Li and X. Chen, Biomaterials, 2010, 31, 3016-3022.

4 M. Yang, K. Cheng, S. Qi, H. Liu, Y. Jiang, H. Jiang, J. Li, K. Chen, H. Zhang and Z. Cheng, Biomaterials, 2013, 34, 2796-2806.

5 K. Cheng, S. R. Kothapalli, H. Liu, A. L. Koh, J. V. Jokerst, H. Jiang, M. Yang, J. Li, J. Levi, J. C. Wu, S. S. Gambhir and Z. Cheng, J. Am. Chem. Soc., 2014, 136, 3560-3571.

6 K. Cheng, M. Yang, R. Zhang, C. Qin, X. Su and Z. Cheng, ACS Nano, 2014, 8, 9884-9896.

7 R. Zhang, K. Cheng, A. L. Antaris, X. Ma, M. Yang, S. Ramakrishnan, G. Liu, A. Lu, H. Dai, M. Tian and Z. Cheng, Biomaterials, 2016, 103, 265-277.

8 H. Y. Lee, Z. Li, K. Chen, A. R. Hsu, C. Xu, J. Xie, S. Sun and X. Chen, J. Nucl. Med., 2008, 49, 1371-1379.

9 Z. Ali, A. Z. Abbasi, F. Zhang, P. Arosio, A. Lascialfari, M. F. Casula, A. Wenk, W. Kreyling, R. Plapper, M. Seidel, R. Niessner, J. Knoll, A. Seubert and W. J. Parak, Anal. Chem., 2011, 83, 2877-2882.

10 P. Laverman, C. A. D'Souza, A. Eek, W. J. McBride, R. M. Sharkey, W. J. Oyen, D. M. Goldenberg and O. C. Boerman, Tumor Biol., 2012, 33, 427-434.
11 X. Sun, W. Cai and X. Chen, Acc. Chem. Res., 2015, 48, 286294.

12 Y. Sun, M. Yu, S. Liang, Y. Zhang, C. Li, T. Mou, W. Yang, X. Zhang, B. Li, C. Huang and F. Li, Biomaterials, 2011, 32, 2999-3007.

13 M. Sarparanta, E. Makila, T. Heikkila, J. Salonen, E. Kukk, V. P. Lehto, H. A. Santos, J. Hirvonen and A. J. Airaksinen, Mol. Pharm., 2011, 8, 1799-1806.

14 J. Zhou, M. Yu, Y. Sun, X. Zhang, X. Zhu, Z. Wu, D. Wu and F. Li, Biomaterials, 2011, 32, 1148-1156.

15 W. G. Kreyling, A. M. Abdelmonem, Z. Ali, F. Alves, M. Geiser, N. Haberl, R. Hartmann, S. Hirn, D. J. de Aberasturi, K. Kantner, G. Khadem-Saba, J.-M. Montenegro, J. Rejman, T. Rojo, I. R. de Larramendi, R. Ufartes, A. Wenk and W. J. Parak, Nat. Nanotechnol., 2015, 10, 619-623.

16 W. J. McBride, R. M. Sharkey, H. Karacay, C. A. D’Souza, E. A. Rossi, P. Laverman, C. H. Chang, O. C. Boerman and D. M. Goldenberg, J. Nucl. Med., 2009, 50, 991-998.

17 S. Liu, H. Liu, H. Jiang, Y. Xu, H. Zhang and Z. Cheng, Eur. J. Nucl. Med. Mol. Imaging, 2011, 38, 1732-1741.

18 P. Laverman, W. J. McBride, R. M. Sharkey, A. Eek, L. Joosten, W. J. Oyen, D. M. Goldenberg and O. C. Boerman, J. Nucl. Med., 2010, 51, 454-461.

19 S. Sun and H. Zeng, J. Am. Chem. Soc., 2002, 124, 8204-8205. 20 M. Persson, H. Liu, J. Madsen, Z. Cheng and A. Kjaer, Nucl. Med. Biol., 2013, 40, 618-624.

21 Z. Miao, G. Ren, H. Liu, L. Jiang and Z. Cheng, J. Biomed. Opt., 2010, 15, 036007.

22 Z. Miao, G. Ren, H. Liu, L. Jiang and Z. Cheng, Bioconjugate Chem., 2010, 21, 947-954. 